\title{
Um Estudo Sobre o Perfil de Professores do Ensino Fundamental e o Uso de Tecnologias para a Educação: uma Proposição de Agenda de Pesquisa a partir de Dados Educacionais
}

\author{
Giovanni Bohm Machado - PPGIE UFRGS - giobohm@terra.com.br \\ Igor Kuhn - PPGIE UFRGS - kuhnigor@gmail.com \\ Francisco Dutra dos Santos Jr. - PPGIE UFRGS - prof.chicosantosjr@gmail.com \\ Leandro Krug Wives - PPGIE UFRGS - leandro.wives@ufrgs.br
}

\begin{abstract}
Resumo. Este artigo busca mapear o perfil dos professores de ensino fundamental em relação à suas dificuldades, preferências, conhecimentos e tendências no uso das tecnologias digitais com o intuito de desenvolver alternativas concretas para a qualificação das ações desenvolvidas por esses professores em suas escolas, utilizando a tecnologia disponivel para este fim. Foi aplicada uma pesquisa de levantamento com professores da rede municipal de ensino da região metropolitana de Porto Alegre, $R S$, onde se obteve uma amostra de 140 respondentes. Os principais resultados indicaram uma forte disposição dos professores em utilizar tecnologias para o ensino, o que proporcionou a geração de uma agenda de pesquisa para aprofundamento do tema.
\end{abstract}

Palavras-chave: formação continuada, tecnologias da informação e comunicação, ensino fundamental, perfil de professores.

\section{A Study about Elementary School Teachers and the Use of Technologies for Education: a Proposition of Research Agenda from Educational Data}

\begin{abstract}
In this paper, we seek to map the profile of primary school teachers about their difficulties, preferences, knowledge, and tendencies in the use of digital technologies to develop concrete alternatives for the qualification of the actions developed by these teachers in their schools using the technology available for this purpose. A survey was conducted with some teachers from the municipal education network in the metropolitan region of Porto Alegre, RS, Brazil, where a sample of 140 respondents was obtained. The main results indicated a strong willingness of the teachers to use technologies for teaching, which provided the generation of a research agenda to deepen the theme.
\end{abstract}

Keywords: continuing education, information and communication technologies, elementary education, teacher's profile.

\section{Introdução}

A tecnologia da informação cresce e difunde-se exponencialmente, modificando totalmente a interação do ser humano com todas as formas de comunicação (Castells, 1999), inclusive aquelas relacionadas ao aprendizado. Nas últimas décadas, o uso da tecnologia por professores tem despertado o interesse de pesquisadores como Gómez (2015) que, em seu livro "Educação na Era Digital: a escola educativa", afirma que a capacidade para usar a tecnologia da informação é cada dia mais decisiva, já que muitos 
dos serviços, do trabalho e dos intercâmbios estão e estarão cada vez mais acessíveis apenas por meio da rede.

No âmbito da educação, as Tecnologias da Informação e Comunicação (TICs) têm assumido um importante papel enquanto ferramentas pedagógicas que podem potencializar os processos de ensino e de aprendizagem. Ainda sobre as TICs, Martha (2013) afirma que as tecnologias de informação e comunicação atuais fomentam uma constante necessidade de superação do saber, fazendo com que se trilhe novos caminhos para a dinâmica entre alunos e professores. Assim, o protagonismo da tecnologia no desenvolvimento dos profissionais da educação pode aprimorar o processo de formação continuada qualificando as práticas pedagógicas no contexto atual (Meirinhos, 2006).

Dados publicados pelo Centro Regional de Estudos para o Desenvolvimento da Sociedade da Informação, Cetic.br (2016), traduzem a necessidade emergente de pesquisas das TIC em âmbito Educacional numa perspectiva pragmática. O mesmo dossiê remete como princípio a garantia de uma educação inclusiva, equitativa e de qualidade, bem como promover oportunidades de aprendizagem para todos ao longo da vida.

Para o profissional da educação, a formação continuada é um processo essencial nesse ambiente de dinâmicas e constantes transformações tecnológicas e sociais. O uso de recursos computacionais é uma realidade, não podendo ser ignorada pelo professor, cujas competências, habilidades e atitudes podem desenvolver-se a partir da ação pedagógica no dia a dia, através do descobrimento e da experimentação dessas novas tecnologias (Moresco e Behar, 2013).

Em estudo realizado por Machado (2013) com professores de ensino fundamental da rede pública de um município da região metropolitana de Porto Alegre foi possível elencar algumas dificuldades enfrentadas por esses professores. Dentre as questões apontadas, destacam-se o pouco tempo destinado para as práticas formativas, a elevada carga horária em sala de aula e os assuntos e necessidades não contemplados pela formação do professor.

Dessa forma, entende-se que a utilização de computadores e dispositivos digitais possa contribuir para minimizar essas dificuldades. Para isso, faz-se necessário, através da mineração de dados em um ou mais banco de dados, traçar um perfil mais detalhado desses professores, verificando suas dificuldades, preferências, conhecimentos e tendências no uso das tecnologias digitais. O mapeamento dessas informações contribui para que, no futuro, novas ferramentas tecnológicas possam ser desenvolvidas para auxiliar professores, otimizando e tornando o trabalho mais assertivo, conectado com os anseios de seus alunos e da sociedade. Através desse perfil, será possível traçar uma agenda de pesquisa para a proposição de alternativas concretas que visam a qualificação das ações desenvolvidas por professores em suas escolas, utilizando as tecnologias digitais disponíveis para esse fim.

Um dos grandes objetivos deste projeto é a constituição de um banco de dados com informações que, no futuro, possam ajudar a traçar um perfil detalhado de professores para aprimorar o seu processo de formação continuada.

Este documento está organizado da seguinte forma. Primeiramente, são apresentados conceitos e fundamentos relacionados com a pesquisa realizada e os métodos executados. Em seguida, são apresentados e analisados dados referentes à relação dos professores com a tecnologia e seus desdobramentos em sua prática de ensino. Posteriormente, são abordadas as fragilidades relativas ao tema de pesquisa, bem como uma proposta de agenda de pesquisa, seguido das considerações finais. 


\section{Fundamentação Teórica}

O contexto da escola e da educação no mundo contemporâneo implica a mobilização de saberes plurais (Tardif, 2002) e exige dos profissionais da educação uma gama de competências também diversa. Hoje, pensar a profissão docente necessita de um olhar que contemple tanto as questões de conteúdo quanto as relacionais em um cenário que se modifica o tempo todo, e que, por isso, precisa estar fortemente vinculado às questões coletivas que pressupõem a socialização e o compartilhamento de saberes.

Dessa forma, os processos formativos precisam estar profundamente comprometidos com os contextos de atuação profissional (Nóvoa, 2002), considerando o protagonismo docente nesses processos, bem como trazendo ao foco a discussão sobre questões cotidianas, relacionadas aos processos de ensino e de aprendizagem. Além disso, a formação precisa propor dinâmicas que ajudem os professores a construírem competências que os permita reinventar soluções e alternativas às situações emergentes, que muitas vezes são inéditas à sua experiência (Sacristán, 1991).

Os processos formativos são também processos de aprendizagem. A partir dessa premissa, é importante pensar a formação docente como um processo que se desenvolve durante toda a trajetória profissional e que vai se moldando de acordo com as experiências dos sujeitos (Nóvoa, 2002; Tardif, 2002). Dessa forma, para pensar a inserção dos profissionais em processos formativos, é condição indispensável compreender quais as competências necessárias, tanto para desempenhar suas funções docentes quanto para estar permanentemente em formação, além de pensar também sobre quais seriam as competências necessárias aos docentes para que consigam participar de processos formativos com o uso das tecnologias.

\section{Metodologia}

No intuito de se obter alinhamento com os objetivos propostos, a estratégia utilizada para este estudo foi a pesquisa de levantamento, também conhecida como survey. De acordo com Gil (2010), ela permite ao pesquisador conhecer comportamentos, opiniões ou características diretamente das pessoas que compõem determinada população a ser estudada. Especificamente neste estudo, a pesquisa de levantamento tem caráter exploratório e descritivo.

A ferramenta utilizada para a coleta de Dados Educacionais (DE) constituiu-se de um questionário estruturado, composto por 31 questões, contendo escalas de múltipla escolha, mistas e do tipo Likert de 5 pontos. Das 31 questões, 11 possuem características sociodemográficas para melhor caracterização da amostra, o que é extremamente importante na formação do perfil dos docentes. As demais questões buscam medir o conhecimento dos docentes, suas preferências e opiniões em relação à utilização de computadores e/ou dispositivos digitais para o exercício de sua profissão.

O questionário foi estruturado a partir da literatura existente e baseado no modelo TAM (Technology Acceptance Model), proposto por Davis (1989), cujo objetivo é fornecer explicação das causas determinantes da aceitação dos computadores. Da mesma forma, visa explicar o comportamento dos usuários em face às diversas tecnologias ligadas à informática, explicando por que um sistema, em particular, pode ser aceito ou invalidado, assim como dando orientações para as devidas correções.

A população-alvo do estudo foi constituída de professores de ensino fundamental das redes de ensino municipal de cidades da região metropolitana da grande Porto Alegre, 
como Canoas, Alvorada, Gravataí e Sapucaia do Sul. Os questionários foram enviados através da ferramenta Google Forms, na segunda quinzena de maio de 2018, tendo como resultado uma amostra 140 respondentes, sendo na sua totalidade professores das redes municipais de ensino.

Alguns testes estatísticos, para verificação de confiabilidade do instrumento de coleta de dados, foram realizados utilizando o software SPSS Statistics. Foi utilizado o teste de confiabilidade Alpha de Cronbach, cujo resultado foi de 0,811 . O resultado está acima do recomendado pela literatura, que é de 0,7 (Hair et al., 2009), o que comprova a significância estatística da escala criada para o questionário.

A normalidade dos dados foi verificada pela análise dos valores de curtose e assimetria (Hair et al., 2009). Segundo Kline (1998), os valores indicados para curtose devem ser abaixo de 10, e os valores de assimetria correspondentes devem permanecer abaixo de 3 . Os resultados acima desses valores indicados podem configurar distribuições problemáticas, não representando distribuições normais. Os resultados obtidos nas questões formuladas ficaram dentro do recomendado pela literatura. Os DE e sua análise podem ser verificados na seção seguinte.

\section{Dados Educacionais e análise}

A aplicação dos questionários permitiu a obtenção de dados significativos relacionados ao perfil dos professores de ensino fundamental das redes de ensino municipal de cidades da região metropolitana de Porto Alegre.

\subsection{Perfil do professor}

Os dados da amostra indicam uma forte predominância do sexo feminino entre os professores consultados, com $84 \%$. O restante, 16\%, são do sexo masculino. Em relação à rede de ensino onde são docentes, $54 \%$ dos professores são oriundos da cidade de Canoas, sendo $11 \%$ de Porto Alegre, $11 \%$ de Alvorada e os restantes divididos entre Sapucaia do Sul, Gravataí e outras cidades. Com relação à faixa etária, 43\% estão localizados entre 30 e 39 anos, $24 \%$ entre 40 e 49 anos, $19 \%$ entre 50 e 59 anos, $10 \%$ entre 25 e 29 anos, $3 \%$ com 60 anos ou mais e apenas $1 \%$ entre 18 e 24 anos. Não houve incidência de professores com menos de 18 anos, conforme indica a Tabela 1.

Tabela 1. Caracterização da amostra

\begin{tabular}{|c|c|c|c|c|c|}
\hline \multirow{7}{*}{ 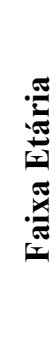 } & Até 18 anos & $0 \%$ & \multirow{6}{*}{ 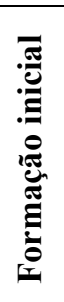 } & & \multirow{2}{*}{$25 \%$} \\
\hline & 18 a 24 anos & $1 \%$ & & Magisterio & \\
\hline & 25 a 29 anos & $10 \%$ & & \multirow{2}{*}{ Licenciatura } & \multirow{2}{*}{$63 \%$} \\
\hline & 30 a 39 anos & $43 \%$ & & & \\
\hline & 40 a 49 anos & $24 \%$ & & \multirow{2}{*}{ Bacharelado } & \multirow{2}{*}{$12 \%$} \\
\hline & 50 a 59 anos & $19 \%$ & & & \\
\hline & 60 anos ou mais & $3 \%$ & \multirow{7}{*}{ 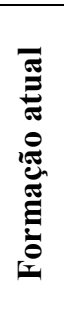 } & & \\
\hline \multirow{2}{*}{ 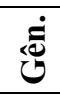 } & Feminino & $84 \%$ & & Especialização & $63 \%$ \\
\hline & Masculino & $16 \%$ & & & \\
\hline \multirow{4}{*}{ 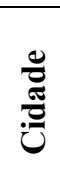 } & Alvorada & $11 \%$ & & Mestrado & $19 \%$ \\
\hline & Canoas & $54 \%$ & & \multirow{2}{*}{ Doutorado } & \multirow{2}{*}{$3 \%$} \\
\hline & Porto Alegre & $11 \%$ & & & \\
\hline & Outras cidades & $27 \%$ & & Não possui & $14 \%$ \\
\hline
\end{tabular}


No que se refere à formação inicial, $63 \%$ dos professores indicam possuir licenciatura, $25 \%$ magistério e $12 \%$ bacharelado. No que diz respeito à formação atual, $63 \%$ possui especialização, $19 \%$ mestrado e apenas 3\% doutorado. Um percentual significativo de $14 \%$ dos respondentes não avançou em sua formação, permanecendo com sua a formação inicial. Dentre este último número, $76 \%$ com licenciatura e $24 \%$ com magistério.

\subsection{Relação do professor com a tecnologia}

Esta etapa trata principalmente sobre como os professores percebem a importância da tecnologia e a sua influência no trabalho exercido por eles. A partir da análise dos dados contidos na Tabela 2, é possível verificar que a tecnologia faz parte do dia a dia dos professores, possuindo um papel significativo em relação à suas atividades. De acordo com Canário (2006), Sacristán (2007) e Bauman (2009), a contemporaneidade traz consigo a necessidade de ressignificação da escola e das relações que nelas se estabelecem. Nesse sentido, segundo os mesmos autores, os modelos, que até então respondiam às demandas do mundo, encontram-se ultrapassados e descolados das necessidades e contextos em que vivemos. Os dados obtidos na pesquisa comprovam que a tecnologia vem promovendo a quebra de paradigma nas antigas relações estabelecidas entre os sujeitos, com o conhecimento e com as formas de aprender.

Tabela 2. Questões principais sobre o perfil do professor na área de estudo

\begin{tabular}{|l|c|c|c|c|c|}
\hline Questão & DF & D & I & CF \\
\hline $\begin{array}{l}\text { Costumo utilizar computadores e/ou dispositivos digitais (tablets, } \\
\text { smartphones) para preparar minhas aulas. }\end{array}$ & $1 \%$ & $3 \%$ & $14 \%$ & $18 \%$ & $65 \%$ \\
\hline Computadores e/ou dispositivos digitais facilitam meu trabalho. & $1 \%$ & $0 \%$ & $7 \%$ & $18 \%$ & $75 \%$ \\
\hline $\begin{array}{l}\text { A utilização de computadores e/ou dispositivos digitais torna meu } \\
\text { trabalho mais prazeroso. }\end{array}$ & $1 \%$ & $4 \%$ & $14 \%$ & $19 \%$ & $62 \%$ \\
\hline $\begin{array}{l}\text { A utilização de computadores e/ou dispositivos digitais aumentou meu } \\
\text { desempenho na escola onde trabalho. }\end{array}$ & $0 \%$ & $3 \%$ & $24 \%$ & $29 \%$ & $44 \%$ \\
\hline $\begin{array}{l}\text { A utilização de computadores e/ou dispositivos digitais melhorou a } \\
\text { qualidade dos materiais educacionais produzidos na escola onde } \\
\text { trabalho. }\end{array}$ & $2 \%$ & $3 \%$ & $19 \%$ & $31 \%$ & $46 \%$ \\
\hline $\begin{array}{l}\text { Utilizo computadores e/ou dispositivos digitais para buscar conteúdos } \\
\text { acadêmicos e profissionais de meu interesse através da internet. }\end{array}$ & $1 \%$ & $1 \%$ & $4 \%$ & $15 \%$ & $79 \%$ \\
\hline $\begin{array}{l}\text { A Internet e a utilização de computadores e/ou dispositivos digitais têm } \\
\text { um papel importante no processo de formação contínua de professores. }\end{array}$ & $1 \%$ & $2 \%$ & $5 \%$ & $18 \%$ & $75 \%$ \\
\hline $\begin{array}{l}\text { Gostaria de receber, através de computadores e/ou dispositivos digitais, } \\
\text { recomendação/indicação de conteúdo, materiais, artigos, relatos, mídias } \\
\text { e demais informações sobre a área em que atuo ou tenho interesse. }\end{array}$ & $2 \%$ & $3 \%$ & $6 \%$ & $22 \%$ & $68 \%$ \\
\hline
\end{tabular}

Legenda: DF - Discordo fortemente $\mid \mathbf{D}$ - Discordo $\mid \mathbf{I}$ - Indiferente $\mid \mathbf{C}$ - Concordo $\mid \mathbf{C F}$ - Concordo fortemente

O uso de computadores e dispositivos digitais, aliado ao uso da Internet, tem melhorado significativamente a quantidade e a qualidade dos materiais educacionais 
produzidos, bem como a dinâmica de interação entre professor e alunos. De acordo com os dados obtidos, $83 \%$ dos professores indicaram que costumam utilizar computadores e/ou dispositivos móveis para preparar suas aulas. Os professores também apontaram que essas tecnologias facilitam seu trabalho no dia a dia (93\%) e costumam tornar seu trabalho mais prazeroso $(81 \%)$.

Ainda sobre os percentuais encontrados, $73 \%$ dos professores indicaram que seu desempenho melhorou na escola onde trabalha e $77 \%$ desses atribuem aos computadores e dispositivos móveis uma melhoria dos materiais educacionais produzidos na escola.

Em relação à busca de conteúdos acadêmicos e profissionais, 94\% realizam essa procura tendo como fonte a Internet e $90 \%$ gostariam de receber materiais de seu interesse e/ou área que atuam. Com relação à formação continuada de professores, $93 \%$ dos respondentes acreditam que a Internet e o uso de computadores e dispositivos digitais têm importante papel nesse processo.

Dentro dessa relação dos professores com a tecnologia, um dado importante evidencia-se em relação aos demais. Quando questionados sobre seu aprendizado na utilização de computadores e dispositivos digitais, $42 \%$ dos professores indicaram ser autodidatas, seguidos de 34\% que receberam ajuda de familiares, amigos ou colegas. Somente $24 \%$ realizam algum curso e/ou treinamento, o que pode estar relacionado à falta de investimento da instituição em relação à formação de seus professores. Esse dado também pode estar associado aos resultados do estudo promovido por Machado (2013), o qual informa sobre o pouco tempo destinado pelas redes municipais de ensino aos professores para realização das práticas formativas, aliado à elevada carga horária em sala de aula. Dessa forma, não parece existir políticas sólidas para promover formação adequada aos professores no uso da tecnologia.

Outro dado relevante é a quantidade de horas diárias em que os professores utilizam a Internet. A grande maioria, 45\%, utilizam a Internet por mais de 4 horas diárias. Esses dados confirmam resultados apresentados pelo WAS (2016), em que coloca o Brasil como o país líder no ranking de tempo conectado a Internet, com uma média de 5 horas/dia por usuário do serviço.

Tabela 3. Perfil tecnológico do professor

\begin{tabular}{|c|c|c|}
\hline Questão & Alternativa & $\%$ \\
\hline \multirow{3}{*}{$\begin{array}{l}\text { Aprendi a utilizar computadores e/ou } \\
\text { dispositivos digitais de forma... }\end{array}$} & Autodidata & $42 \%$ \\
\hline & Curso e/ou treinamento & $24 \%$ \\
\hline & Com ajuda de familiares, amigos ou colegas & $34 \%$ \\
\hline \multirow{5}{*}{$\begin{array}{l}\text { Em média, quanto tempo por dia você } \\
\text { costuma utilizar computadores e/ou } \\
\text { dispositivos digitais? }\end{array}$} & Menos de 1 hora & $1 \%$ \\
\hline & 1 a 2 horas & $14 \%$ \\
\hline & 2 a 3 horas & $20 \%$ \\
\hline & 3 a 4 horas & $19 \%$ \\
\hline & Mais de 4 horas & $45 \%$ \\
\hline \multirow{4}{*}{$\begin{array}{l}\text { Quais dispositivos digitais você utiliza } \\
\text { para preparar suas aulas? }\end{array}$} & Desktop & $16 \%$ \\
\hline & Notebook & $46 \%$ \\
\hline & Smartphone & $31 \%$ \\
\hline & Tablet & $7 \%$ \\
\hline
\end{tabular}

Talvez o resultado que se destaca da pesquisa tenha sido a informação de quais dispositivos os professores mais utilizam para preparar suas aulas. A utilização de tablets e smartphones alcançaram, somados, $38 \%$ dos respondentes, conforme pode ser observado na Tabela 3. Dada a dificuldade no manuseio e pequenas dimensões dos 
referidos dispositivos em relação aos desktops e notebooks, esse dado pode ser considerado surpreendente. $\mathrm{O}$ estudo indica uma utilização mais intensiva da tecnologia pelos professores que parecem estar aderindo-a não só em sala de aula, mas também na preparação das aulas. É preciso aprofundar a pesquisa a fim de descobrir, no entanto, de que forma esses dispositivos estão sendo utilizados efetivamente para preparação das aulas. De que forma se dá essa utilização? Os professores estão pesquisando conteúdos para as aulas, preparando materiais educacionais, apresentações ou outros? Essas perguntas necessitam de maior esclarecimento. Sacristán (1991) afirma que a uma boa formação faz com que professores construam competências que podem reinventar a forma como se dá a dinâmica em sala de aula, construindo novas maneiras de relacionamento, inovando nas práticas pedagógicas. Dessa forma, é imperativo que essas questões sejam realmente melhor investigadas para que seja possível inovar no ensino através da utilização da tecnologia.

\section{Proposta de agenda de pesquisa}

Os países que optaram pelo desenvolvimento econômico, entre eles o Brasil, definiram no âmbito de suas políticas públicas elaborar Políticas de Tecnologia Educacional a partir de metas definidas em orçamentos plurianuais (Brasil, 2014; Dellagnelo, 2015). Foram rigorosos na execução a curto, médio e longo prazo. Atribuíram o conceito de Planejamento Multidimensional, o qual propõe como condição a participação simultânea de fatores humanos e de infraestrutura, onde os elementos da condição humana pressupõem perspectivas como competências de gestores e professores em relação à infraestrutura, à necessidade de recursos pedagógicos, além de equipamentos e conexão à Internet.

Nesse contexto, vamos definir aqui o conceito de competência utilizado, que é o quanto gestores e professores conhecem e sentem-se confortáveis com uso de tecnologia para fins pedagógicos. Destacamos que essa abordagem se fundamenta na Teoria Quatro Dimensões desenvolvida pelo Centro de Inovação Educacional da Holanda, a qual o Centro de Inovação para Educação Brasileira (CIEB) tem adotado como marco conceitual do seu trabalho para análise e proposições de políticas públicas em relação às tecnologias educacionais no Brasil (Dellagnelo, 2015).

Ao traçar um perfil do professor de ensino fundamental de escolas da região metropolitana de Porto Alegre, foi possível detectar alguns pontos nos quais se propõe uma agenda de pesquisa para aprofundamento de algumas das questões levantadas. No futuro, com a inserção de um maior volume de dados no banco, a utilização da mineração de dados será ainda melhor explorada para o aprimoramento desta agenda a partir de suas descobertas. Cabe ainda a sugestão de adoção de políticas públicas para melhoria das condições de trabalho dos professores e a consequente busca pela excelência no ensino, conforme Quadro 1.

Para que possam ser colocadas em prática, uma pesquisa mais extensa e completa envolvendo um quadro mais representativo dos professores em um âmbito nacional é necessária. Além disso, faz-se pertinente a aplicação de modelos e algoritmos de análise analítica de dados (learning analytics), contextualizados nos achados e indicadores aqui descritos.

Quadro 1. Proposição de agenda de pesquisa e políticas públicas através da mineração de dados 


\begin{tabular}{|l|l|}
\hline Proposição & Ação \\
\hline $\begin{array}{l}\text { Relacionar a faixa-etária, gênero, formação dos professores e a utilização de tecnologias } \\
\text { no ensino; }\end{array}$ & Pesquisa \\
\hline $\begin{array}{l}\text { Investigar o impacto da utilização de computadores e dispositivos digitais no } \\
\text { desempenho dos professores e dos alunos em sala de aula; }\end{array}$ & Pesquisa \\
\hline $\begin{array}{l}\text { Identificar de que forma os professores utilizam smartphones e tablets para preparação } \\
\text { de suas aulas e qual o impacto disso na aprendizagem de seus alunos; }\end{array}$ & Pesquisa \\
\hline $\begin{array}{l}\text { Levantar e avaliar as dificuldades encontradas pelos professores nas redes e escolas em } \\
\text { que trabalham em relação ao uso de tecnologias para educação; }\end{array}$ & Pesquisa \\
\hline $\begin{array}{l}\text { Investigar se o uso de aplicativos para dispositivos móveis, operando através de uma } \\
\text { rede colaborativa de professores e profissionais do ensino, pode-se constituir em uma } \\
\text { ferramenta efetiva para facilitar e aprimorar o processo de formação continuada de } \\
\text { professores; }\end{array}$ & Pesquisa \\
\hline $\begin{array}{l}\text { Implementação de políticas públicas para fomento da formação continuada de } \\
\text { professores, tais como bolsas de estudos, licenças remuneradas entre outros programas; }\end{array}$ & $\begin{array}{l}\text { Políticas } \\
\text { públicas }\end{array}$ \\
\hline $\begin{array}{l}\text { Investimento em treinamento e capacitações para uso de ferramentas tecnológicas por } \\
\text { parte do professor. }\end{array}$ & $\begin{array}{l}\text { Políticas } \\
\text { públicas }\end{array}$ \\
\hline
\end{tabular}

\section{Considerações Finais}

A pesquisa realizada cuja principal fonte de dados foram os professores da rede municipal de ensino de cidades da região metropolitana de Porto Alegre forneceu uma base de dados educacionais capaz de traçar um perfil desses profissionais, proporcionando a possibilidade de geração de novos estudos e a consequente criação de novas ferramentas tecnológicas capazes de aprimorar o trabalho docente.

A inserção de tecnologias no ambiente escolar pode contribuir para expandir o acesso à informação e promover a criação de comunidades colaborativas de aprendizagem que privilegiam a construção do conhecimento, a comunicação e a formação dos professores (Almeida e Rubim, 2001), fazendo com que os docentes tenham maior oportunidades e facilidade de aprimorar seus conhecimentos, podendo tornar sua atividade muito mais efetiva.

As informações prestadas pelos professores, alvos deste estudo, contribuíram para a geração de uma agenda de pesquisas capaz de elucidar algumas questões ainda obscuras nos estudos que tratam da influência da tecnologia na atividade docente. Essa agenda proposta não é definitiva, mas pode sinalizar um avanço nos estudos sobre o tema, além de oportunizar a geração de novas ferramentas tecnológicas por parte de desenvolvedores, o qual podem utilizar as propostas como ponto de partida para seus próximos projetos. A criação de novas ferramentas tecnológicas poderá contribuir para as práticas formativas dos professores, proporcionando maior possibilidade de se obter excelência no processo de ensino e aprendizagem realizado por esses profissionais. Através da coleta de dados em grande escala é possível criar modelos e fazer predições que serão aplicáveis em qualquer ambiente virtual de aprendizagem e até mesmo em salas de aula convencionais (Baker; Isotani; Carvalho, 2011), o que pode promover o melhoramento na oferta de cursos de extensão, treinamentos e outras atividades de ensino que contribuam para a formação continuada de professores.

Ainda como proposta de trabalhos futuros, cabe o aprofundamento de alguns elementos observados durante a construção deste estudo, tais como a utilização de dispositivos móveis para preparação de materiais para as aulas, o modo como os 
professores aprendem a utilizar computadores e dispositivos móveis, e o baixo percentual de professores que realizam cursos de capacitação e treinamento. Cabe ainda observar a extensa agenda de pesquisa que o tema nos proporciona, cabendo ainda como proposta de pesquisa o desenvolvimento e aplicação de questionários agrupados por temáticas, aplicados no mesmo grupo alvo estudado no presente artigo.

\section{Referências}

ALMEIDA, M. E. B. de; RUBIM, L. C. B. Tecnologia de informação e comunicação na escola: aprendizagem e produção da escrita. PUC-SP. Disponível em http://www.eadconsultoria.com.br/matapoio/biblioteca/textos_pdf/texto24.pdf. Acessado em 10/04/2018, 2001.

BAKER, Ryan; ISOTANI, Seiji; Carvalho, Adriana. Mineração de Dados Educacionais: Oportunidades para o Brasil. Revista Brasileira de Informática na Educação, [s. 1.], v. 19, n. 02, p. 3-13, 2011.

BAUMAN, Z. Entrevista sobre educação, desafios pedagógicos e modernidade líquida. Cadernos de Pesquisa. v.39, n.137, pp. 661-684, 2009.

BEHAR, P. A. Competências em Educação a Distância. Porto Alegre: Artmed, 2013.

BRASIL. Plano Nacional de Educação 2014-2024 [recurso eletrônico]: Lei $n^{\circ}$ 13.005, de 25 de junho de 2014. Brasília: Câmara dos Deputados, Edições Câmara, 2014.

CANÁRIO, R. A escola tem futuro? Das promessas às incertezas. Porto Alegre: Artmed, 2006.

CASTELLS, M. A Era da Informação: Economia, Sociedade e Cultura, v.1 - O Poder da Identidade. São Paulo: Ed. Paz e Terra, 1999.

CETIC.BR. Centro Regional de Estudos para o Desenvolvimento da Sociedade da Informação. Disponível em: http://www.cetic.br/media/docs/publicacoes/2/ TIC_EDU_2016_LivroEletronico.pdf. Acessado em 10/04/2018, 2016.

GIL, A. C. Como elaborar projetos de pesquisa. São Paulo: Atlas, 2010.

GÓMEZ, A. I. P. Educação na Era Digital: a escola educativa. Tradução Marisa Guedes. Porto Alegre, Penso, 2015.

DAVIS, F. D. Perceived usefulness, perceived ease of use, and user acceptance of information technology. MIS Quarterly, v.13, n., pp. 319-341, 1989.

HAIR, J. F.; BABIN, B.; MONEY, A.; SAMOUEL, P. Fundamentos de métodos de Pesquisa em administração. Porto Alegre: Bookman, 2005.

KLEIN, A. Z.; SILVA, L. V; MACHADO, L.; AZEVEDO, D. Metodologia de Pesquisa em Administração: uma abordagem prática. São Paulo: Atlas, 2015.

LAKATOS, E. M.; MARCONI, M. A. Fundamentos de metodologia científica: técnicas de pesquisa, 6 ed. São Paulo: Atlas, 2009.

MARTHA, G. Educ@r - A (r)evolução Digital na Educação. São Paulo: Ed Saraiva, 2013.

MACHADO, Juliana Aquino. A escola como espaço de formação continuada de professores: um estudo no contexto da rede municipal de ensino de Canoas - RS. 
Dissertação (Mestrado em Educação). UNILASALLE. Programa de Pós-Graduação em Educação. Canoas, 2013.

MEIRINHOS, M. F. A. Desenvolvimento profissional docente em ambientes colaborativos de aprendizagem a distância: estudo de caso no âmbito da formação contínua. Tese de Doutorado. Universidade do Minho. Portugal, 2006.

NÓVOA, A. Formação de professores e trabalho pedagógico. Lisboa: Educa, 2002.

SACRISTÁN, J. G. Consciência e acção sobre a prática como libertação profissional dos professores. In: NÓVOA, A. Profissão professor. Porto Editora: Portugal, 1991.

SACRISTÁN, J. G. A educação que ainda é possível: ensaios sobre uma cultura para a educação. Porto Alegre: Artmed, 2007.

TARDIF, M. Saberes docentes e formação profissional. Petrópolis, RJ: Vozes, 2002.

WAS, WE ARE SOCIAL. Digital in 2016: special reports. Reino Unido. Disponível em http://wearesocial.com/special-reports/digital-in-2016. Acessado em 19/03/2018, 2016.

YIN, R. K. Estudo de caso: planejamento e métodos. Porto Alegre: Bookman, 2010. 